Pacific

Journal of

Mathematics

COMMUTATORS WITH POWER CENTRAL VALUES ON A LIE IDEAL

Luisa Carini and Vincenzo De Filippis

Volume $193 \quad$ No. 2

April 2000 


\title{
COMMUTATORS WITH POWER CENTRAL VALUES ON A LIE IDEAL
}

\section{Luisa Carini and Vincenzo De Filippis}

\begin{abstract}
Let $R$ be a prime ring of characteristic $\neq 2$ with a derivation $d \neq 0, L$ a noncentral Lie ideal of $R$ such that $[d(u), u]^{n}$ is central, for all $u \in L$. We prove that $R$ must satisfy $s_{4}$ the standard identity in 4 variables. We also examine the case $R$ is a 2-torsion free semiprime ring and $[d([x, y]),[x, y]]^{n}$ is central, for all $x, y \in R$.
\end{abstract}

Let $R$ be a prime ring and $d$ a nonzero derivation of $R$. A well known result of Posner [14] states that if the commutator $[d(x), x] \in Z(R)$, the center of $R$, for any $x \in R$, then $R$ is commutative.

In [11] C. Lanski generalizes the result of Posner to a Lie ideal. To be more specific, the statement of Lanski's theorem is the following:

Theorem $([\mathbf{1 1}$, Theorem 2, page 282]). Let $R$ be a prime ring, $L$ a noncommutative Lie ideal of $R$ and $d \neq 0$ a derivation of $R$. If $[d(x), x] \in Z(R)$, for all $x \in L$, then either $R$ is commutative, or $\operatorname{char}(R)=2$ and $R$ satisfies $s_{4}$, the standard identity in 4 variables.

Here we will examine what happens in case $[d(x), x]^{n} \in Z(R)$, for any $x \in L$, a noncommutative Lie ideal of $R$ and $n \geq 1$ a fixed integer.

One cannot expect the same conclusion of Lanski's theorem as the following example shows:

Example 1. Let $R=M_{2}(F)$, the $2 \times 2$ matrices over a field $F$, and take $L=R$ as a noncommutative Lie ideal of $R$. Since $[x, y]^{2} \in Z(R)$, for all $x, y \in R$, then also $[d(x), x]^{2} \in Z(R)$, for all $x \in R$.

We will prove that:

Theorem 1.1. Let $R$ be a prime ring of characteristic different from $2, L a$ noncentral Lie ideal of $R, d$ a nonzero derivation of $R$ such that $[d(u), u]^{n} \in$ $Z(R)$, for any $u \in L$. Then $R$ satisfies $s_{4}$.

We will proceed by first proving that:

Lemma 1.1. Let $R$ be a prime ring of characteristic different from $2, L$ a noncentral Lie ideal of $R, d$ a nonzero derivation of $R, n \geq 1$. If $d$ satisfies $[d(u), u]^{n}=0$, for any $u \in L$, then $R$ is commutative. 
We then examine the case $R$ is a 2-torsion free semiprime ring. The results we obtain are:

Theorem 2.1. Let $R$ be a 2-torsion free semiprime ring, $d$ a nonzero derivation of $R, n$ a fixed positive integer, $U$ the left Utumi quotient ring of $R$ and $[d([x, y]),[x, y]]^{n}=0$, for any $x, y \in R$. Then there exists a central idempotent element $e$ of $U$ such that on the direct sum decomposition $e U \oplus$ $(1-e) U, d$ vanishes identically on $e U$ and the ring $(1-e) U$ is commutative.

Theorem 2.2. Let $R$ be a 2-torsion free semiprime ring, $d$ a nonzero derivation of $R, n$ a fixed positive integer, $U$ the left Utumi quotient ring of $R$ and $[d([x, y]),[x, y]]^{n} \in Z(R)$, for any $x, y \in R$. Then there exists a central idempotent $e$ of $U$ such that, on the direct sum decomposition $U=e U \oplus(1-e) U$, the derivation d vanishes identically on $e U$ and the ring $(1-e) U$ satisfies $s_{4}$.

\section{The case: $R$ prime ring.}

In all that follows, unless stated otherwise, $R$ will be a prime ring of characteristic $\neq 2, L$ a Lie ideal of $R, d \neq 0$ a derivation of $R$ and $n \geq 1$ a fixed integer such that $[d(x), x]^{n} \in Z(R)$, for all $x \in L$.

For any ring $S, Z(S)$ will denote its center, and $[a, b]=a b-b a,[a, b]_{2}=$ $[[a, b], b], a, b \in S$. In addition $s_{4}$ will denote the standard identity in 4 variables.

We will also make frequent use of the following result due to Kharchenko [8] (see also [12]):

Let $R$ be a prime ring, $d$ a nonzero derivation of $R$ and $I$ a nonzero twosided ideal of $R$. Let $f\left(x_{1}, \ldots, x_{n}, d\left(x_{1}, \ldots, x_{n}\right)\right)$ a differential identity in $I$, that is

$$
f\left(r_{1}, \ldots, r_{n}, d\left(r_{1}\right), \ldots, d\left(r_{n}\right)\right)=0 \quad \forall r_{1}, \ldots, r_{n} \in I .
$$

One of the following holds:

1) Either $d$ is an inner derivation in $Q$, the Martindale quotient ring of $R$, in the sense that there exists $q \in Q$ such that $d=\operatorname{ad}(q)$ and $d(x)=$ $\operatorname{ad}(q)(x)=[q, x]$, for all $x \in R$, and $I$ satisfies the generalized polynomial identity

$$
f\left(r_{1}, \ldots, r_{n},\left[q, r_{1}\right], \ldots,\left[q, r_{n}\right]\right)=0
$$

2) or $I$ satisfies the generalized polynomial identity

$$
f\left(x_{1}, \ldots, x_{n}, y_{1}, \ldots, y_{n}\right)=0 .
$$

Lemma 1.1. Let $R$ be a prime ring of characteristic different from $2, U$ a noncentral Lie ideal of $R, d$ a nonzero derivation of $R$ and $n \geq 1$. If $([d(u), u])^{n}=0$, for any $u \in L$, then $R$ is commutative. 
Proof. Since we assume that $\operatorname{char}(R) \neq 2$, by a result of Herstein [6], $L \supseteq[I, R]$, for some $I \neq 0$, an ideal of $R$, and also $L$ is not commutative. Therefore we will assume throughout that $L \supseteq[I, R]$. Without loss of generality we can assume $L=[I, I]$.

Hence $[d([x, y]),[x, y]]^{n}=0$, for any $x, y \in I$, then $I$ satisfies the differential identity

$$
f(x, y, d(x), d(y))=[[d(x), y]+[x, d(y)],[x, y]]^{n}=0 .
$$

If the derivation $d$ is not inner, by Kharchenko's theorem [8], I satisfies the polynomial identity

$$
f(x, y, t, z)=[[z, y]+[x, t],[x, y]]^{n}=0
$$

and in particular, for $z=0$,

$$
[[x, t],[x, y]]^{n}=0 .
$$

Since the latter is a polynomial identity for $I$, and so for $R$ too, it is well known that there exists a field $F$ such that $R$ and $F_{m}$ satisfy the same polynomial identities (see [7, page 57 , page 89]). Let $e_{i j}$ the matrix unit with 1 in (i,j)-entry and zero elsewhere. Suppose $m \geq 2$. If we choose $x=e_{11}, y=e_{21}, t=e_{12}$, then we get the contradiction

$$
0=\left[\left[e_{11}, e_{12}\right],\left[e_{11}, e_{21}\right]\right]^{n}=\left[e_{12},-e_{21}\right]^{n}=(-1)^{n} e_{11}+e_{22} \neq 0 .
$$

Therefore $m=1$ and so $R$ is commutative.

Let now $d$ be an inner derivation induced by an element $A \in Q$, the Martindale quotient ring of $R$. Then, for any $x, y \in I,\left([A,[x, y]]_{2}\right)^{n}=0$. Since by [2] $I$ and $Q$ satisfy the same generalized polynomial identities, we have $\left([A,[x, y]]_{2}\right)^{n}=0$, for any $x, y \in Q$. Moreover, since $Q$ remains prime by the primeness of $R$, replacing $R$ by $Q$ we may assume that $A \in R$ and $C$ is just the center of $R$. Note that $R$ is a centrally closed prime C-algebra in the present situation [4], i.e., $R C=R$. By Martindale's theorem in [13], $R C$ (and so $R$ ) is a primitive ring which is isomorphic to a dense ring of linear transformations of a vector space $V$ over a division ring $D$. Since $R$ is primitive then there exist a vector space $V$ and the division ring $D$ such that $R$ is dense of D-linear transformation over $V$.

Assume first that $\operatorname{dim}_{D} V \geq 3$.

\section{Step 1.}

We want to show that, for any $v \in V, v$ and $A v$ are linearly D-dependent.

Since if $A v=0$ then $\{v, A v\}$ is D-dependent, suppose that $A v \neq 0$. If $v$ and $A v$ are D-independent, since $\operatorname{dim}_{D} V \geq 3$, then there exists $w \in V$ such that $v, A v, w$ are also linearly independent. By the density of $I$, there exist $x, y \in I$ such that

$$
\begin{aligned}
& x v=0, x A v=w, x w=v \\
& y v=0, y A v=0, y w=w .
\end{aligned}
$$


These imply that

$[A,[x, y]]_{2} v=-v$ and $0=\left([A,[x, y]]_{2}\right)^{n} v=(-1)^{n} v$

which is a contradiction.

So we can conclude that $v$ are $A v$ are linearly D-dependent, for all $v \in V$.

\section{Step 2.}

We show here that there exists $b \in D$ such that $A v=v b$, for any $v \in V$. Now choose $v, w \in V$ linearly independent. Since $\operatorname{dim}_{D} V \geq 3$, there exists $u \in V$ such that $v, w, u$ are linearly independent. By Step 1, there exist $a_{v}, a_{w}, a_{u} \in D$ such that

$A v=v a_{v}, A w=w a_{w}, A u=u a_{u}$ that is $A(v+w+u)=v a_{v}+w a_{w}+u a_{u}$.

Moreover $A(v+w+u)=(v+w+u) a_{v+w+u}$, for a suitable $a_{v+w+u} \in D$. Then $0=v\left(a_{v+w+u}-a_{v}\right)+w\left(a_{v+w+u}-a_{w}\right)+u\left(a_{v+w+u}-a_{u}\right)$ and, because $v, w, u$ are linearly independent, $a_{u}=a_{w}=a_{v}=a_{v+w+u}$. This completes the proof of Step 2.

Let now $r \in R$ and $v \in V$. By Step 2, $A v=v b, r(A v)=r(v b)$, and also $A(r v)=(r v) b$. Thus $0=[A, r] v$, for any $v \in V$, that is $[A, r] V=0$. Since $V$ is a left faithful irreducible R-module, $[A, r]=0$, for all $r \in R$, i.e., $A \in Z(R)$ and $d=0$, which contradicts our hypothesis.

Therefore $\operatorname{dim}_{D} V$ must be $\leq 2$. In this case $R$ is a simple GPI ring with 1 , and so it is a central simple algebra finite dimensional over its center. From Lemma 2 in [10] it follows that there exists a suitable field $F$ such that $R \subseteq M_{k}(F)$, the ring of all $k \times k$ matrices over $F$, and moreover $M_{k}(F)$ satisfies the same generalized polynomial identity of $R$.

If we assume $k \geq 3$, by the same argument as in Steps 1 and 2, we get a contradiction.

Obviously if $k=1$ then $R$ is commutative. Thus we may assume $R \subseteq$ $M_{2}(F)$, where $M_{2}(F)$ satisfies $\left([A,[x, y]]_{2}\right)^{n}=0$.

Since for any $a, b \in M_{2}(F),[a, b]^{2} \in Z(R)$ then it follows easily that $\left([A,[x, y]]_{2}\right)^{2}=0$, for any $x, y \in M_{2}(F)$. Let $A=\left[\begin{array}{ll}a_{11} & a_{12} \\ a_{21} & a_{22}\end{array}\right]$. If we choose $x=e_{12}, y=e_{21}$ then we get:

$$
\begin{gathered}
{\left[A, e_{11}-e_{22}\right]_{2}=\left[\begin{array}{cc}
0 & 4 a_{12} \\
4 a_{21} & 0
\end{array}\right]} \\
0=\left(\left[A, e_{11}-e_{22}\right]_{2}\right)^{2}=\left[\begin{array}{cc}
16\left(a_{12} a_{21}\right) & 0 \\
0 & 16\left(a_{12} a_{21}\right)
\end{array}\right] .
\end{gathered}
$$

Therefore either $a_{12}=0$ or $a_{21}=0$. Without loss of generality we can pick $a_{12}=0$. 
Now let $[x, y]=\left[e_{11}, e_{12}+e_{21}\right]=e_{12}-e_{21}$. In this case we have:

$$
\begin{gathered}
{\left[A, e_{12}-e_{21}\right]_{2}=\left[\begin{array}{cc}
2\left(a_{22}-a_{11}\right) & -2 a_{21} \\
-2 a_{21} & 2\left(a_{11}-a_{22}\right)
\end{array}\right]} \\
\left(\left[\begin{array}{cc}
2\left(a_{22}-a_{11}\right) & -2 a_{21} \\
-2 a_{21} & 2\left(a_{11}-a_{22}\right)
\end{array}\right]\right)^{2}=0
\end{gathered}
$$

that is

$$
\begin{aligned}
& 4\left(a_{21}\right)^{2}+4\left(a_{11}-a_{22}\right)^{2}=0 \\
& \left.\left(a_{21}\right)^{2}=-\left(a_{22}-a_{11}\right)^{2} \quad \mathbf{1}\right) .
\end{aligned}
$$

On the other hand if $[x, y]=\left[e_{11}, e_{12}-e_{21}\right]=e_{12}+e_{21}$ then

$$
\begin{gathered}
\left(\left[A, e_{12}+e_{21}\right]_{2}\right)^{2}=\left[\begin{array}{cc}
2\left(a_{11}-a_{22}\right) & -2 a_{21} \\
2 a_{21} & 2\left(a_{22}-a_{11}\right)
\end{array}\right] \\
\left(\left[\begin{array}{cc}
2\left(a_{11}-a_{22}\right) & -2 a_{21} \\
2 a_{21} & 2\left(a_{22}-a_{11}\right)
\end{array}\right]\right)^{2}=0
\end{gathered}
$$

that is

$$
\begin{gathered}
4\left(a_{22}-a_{11}\right)^{2}-4\left(a_{21}\right)^{2}=0 \\
\left(a_{21}\right)^{2}=\left(a_{22}-a_{11}\right)^{2} \quad(\mathbf{2}) .
\end{gathered}
$$

(1) and (2) imply that $a_{21}=0$ and $a_{11}=a_{22}$ which means that $A$ is a central matrix in $M_{2}(F), A \in F$ and $d=0$, a contradiction. Therefore $k=1$, i.e., $R$ is commutative.

Lemma 1.2. Let $R=M_{k}(F)$, the ring of $k \times k$ matrices over a field $F$ of characteristic $\neq 2$. If $q \neq 0$ is a noncentral element of $R$ such that $\left([q,[x, y]]_{2}\right)^{n} \in F$, for any $x, y \in R$, then $k \leq 2$.

Proof. Suppose $k \geq 3$. Let $i, j, r$ be distinct indices and $q=\sum a_{m n} e_{m n}$, with $a_{m n} \in F$. For simplicity we assume that $i=1, j=2, r=3$. If we choose $[x, y]=\left[e_{12}, e_{23}-e_{31}\right]=e_{13}+e_{32}$, then

$$
[q,[x, y]]_{2}=a_{21} e_{11}+a_{21} e_{22}-2 a_{21} e_{33}+\sum_{n \neq 1} \gamma_{n} e_{1 n}+\sum_{m \neq 2} \delta_{m} e_{m 2}
$$

with $\gamma_{n}, \delta_{m} \in F$, and

$$
\left([q,[x, y]]_{2}\right)^{n}=\left(a_{21}\right)^{n} e_{11}+\left(a_{21}\right)^{n} e_{22}+\left(-2 a_{21}\right)^{n} e_{33}+\sum_{n \neq 1} \alpha_{n} e_{1 n}+\sum_{m \neq 2} \beta_{m} e_{m 2}
$$

with $\alpha_{n}, \beta_{m} \in F$. Since by assumption $\left([q,[x, y]]_{2}\right)^{n} \in F$, then $\alpha_{n}=\beta_{m}=0$, for all $m, n$, and $\left(a_{21}\right)^{n}=\left(-2 a_{21}\right)^{n}=0$, i.e., $a_{21}=0$. In a similar way we may conclude that $a_{i j}=0$, for any $i \neq j$. Therefore if $k \geq 3, q$ is a diagonal matrix, $q=\sum_{t} a_{t t} e_{t t}$, with $a_{t} \in F$.

If we show that $q$ is a central matrix, then we get a contradiction to our assumption and so $k$ must be less or equal than 2 . 
Let $[x, y]=\left[e_{i j}-e_{j i}, e_{j j}\right]=e_{i j}+e_{j i}$. Therefore

$$
[q,[x, y]]_{2}=2\left(a_{i i}-a_{j j}\right) e_{i i}+2\left(a_{j j}-a_{i i}\right) e_{j j}
$$

and

$$
\left([q,[x, y]]_{2}\right)^{n}=2^{n}\left(a_{i i}-a_{j j}\right)^{n} e_{i i}+2^{n}\left(a_{j j}-a_{i i}\right)^{n} e_{j j} .
$$

Since $\left([q,[x, y]]_{2}\right)^{n} \in F$ and $k \geq 3$, it follows that $a_{i i}=a_{j j}$. Thus $q$ is a central matrix.

Notice that if $n=1$ then by using the same argument and choosing $[x, y]=e_{12}$, we get $N=[q,[x, y]]_{2}=-2 e_{12} q e_{12}$, which has rank 1 and so it cannot be central in $M_{k}(F)$, with $k \geq 2$. This implies that if $n=1$ then $k=1$, and $R$ must be a commutative field. The proof of Lemma 1.2 is now complete.

Theorem 1.1. Let $R$ be a prime ring of characteristic different from $2, L$ a noncentral Lie ideal of $R, d$ a nonzero derivation of $R$ such that $[d(u), u]^{n} \in$ $Z(R)$, for any $u \in L$. Then $R$ satisfies $s_{4}$.

Proof. Let $I$ be the nonzero two-sided ideal of $R$ such that $0 \neq[I, R] \subseteq L$ and $J$ be any nonzero two-sided ideal of $R$. Then $V=\left[I, J^{2}\right] \subseteq L$ is a Lie ideal of $R$. If, for every $v \in V,[d(v), v]^{n}=0$, by Lemma 1.1, $R$ is commutative. Otherwise, by our assumptions, $J \cap Z(R) \neq 0$. Let now $K$ be a nonzero two-sided ideal of $R_{Z}$, the ring of the central quotients of $R$. Since $K \cap R$ is an ideal of $R$ then $K \cap R \cap Z(R) \neq 0$, that is $K$ contains an invertible element in $R_{Z}$, and so $R_{Z}$ is simple with 1 .

Moreover we may assume $L=[I, I]$. For any $x, y \in I,[d([x, y]),[x, y]]^{n} \in$ $Z(R)$, i.e.,

$$
\left[[d([x, y]),[x, y]]^{n}, r\right]=0 \quad \text { for any } x \in R .
$$

Thus $I$ satisfies the differential identity

$$
f(x, y, r, d(x), d(y))=\left[[[d(x), y]+[x, d(y)],[x, y]]^{n}, r\right]=0 .
$$

If the derivation is not inner, by [8], $I$ satisfies the polynomial identity

$$
f(x, y, r, z, t)=\left[[[t, y]+[x, z],[x, y]]^{n}, r\right]=0
$$

and in particular, for $z=0$,

$$
\left[[[t, y],[x, y]]^{n}, r\right]=0 .
$$

In this case we know that there exists a field $F$ such that $R$ and $F_{m}$ satisfy the same polynomial identities. Thus $[[t, y],[x, y]]^{n}$ is central in $F_{m}$. Suppose $m \geq 3$ and choose $x=e_{32}, y=e_{33}, t=e_{23}$.

$$
\begin{gathered}
{[t, y]=e_{23},[x, y]=-e_{32}} \\
{[[t, y],[x, y]]=-e_{22}+e_{33}} \\
{[[t, y],[x, y]]^{n}=(-1)^{n} e_{22}+e_{33} \notin Z(R)}
\end{gathered}
$$

contrary to our assumptions. This forces $m \leq 2$, i.e., $R$ satisfies $s_{4}$. 
Notice that in the case $n=1,[[t, y],[x, y]]$ must be central in $F_{m}$. But if $m \geq 2$ and $t=e_{11}, y=e_{12}, x=e_{21}$, we get the contradiction $[[t, y],[x, y]]=$ $2 e_{12} \notin Z(R)$. Therefore $m$ must be equal to 1 and $R$ is commutative.

Now let $d$ be an inner derivation induced by an element $A \in Q$. By localizing $R$ at $Z(R)$ it follows that $\left([A,[x, y]]_{2}\right)^{n} \in Z\left(R_{Z}\right)$, for all $x, y \in R_{Z}$.

Since $R$ and $R_{Z}$ satisfy the same polynomial identities, in order to prove that $R$ satisfies $S_{4}\left(x_{1}, x_{2}, x_{3}, x_{4}\right)$, we may assume that $R$ is simple with 1 and $[R, R] \subseteq L$.

In this case, $\left([A,[x, y]]_{2}\right)^{n} \in Z(R)$, for all $x, y \in R$. Therefore $R$ satisfies a generalized polynomial identity and it is simple with 1 , which implies that $Q=R C=R$ and $R$ has a minimal right ideal. Thus $A \in R=Q$ and $R$ is simple artinian that is $R=D_{k}$, where $D$ is a division ring finite dimensional over $Z(R)$ [13]. From Lemma 2 in [10] it follows that there exists a suitable field $F$ such that $R \subseteq M_{k}(F)$, the ring of all $k \times k$ matrices over $F$, and moreover $M_{k}(F)$ satisfies the generalized polynomial identity $\left[\left([A,[x, y]]_{2}\right)^{n}, z\right]=0$. By Lemma 1.2 , if $n \geq 2$ then $k \leq 2$ and $R$ satisfies $s_{4}$, also if $n=1$ then $k=1$ and $R$ must be commutative.

\section{The case: $R$ semiprime ring.}

In all that follows $R$ will be a 2-torsion free semiprime ring. We cannot expect the same conclusion of previous section to hold, as the following example shows:

Example 2. Let $R_{1}$ be any prime ring not satisfying $s_{4}$ and $R_{2}=M_{2}(F)$, the ring of $2 \times 2$ matrices over the field $F$. Let $R=R_{1} \oplus R_{2}, d$ a nonzero derivation of $R$ such that $d=0$ in $R_{1}$. Consider $L=[R, R]$. It is a noncentral Lie ideal of $R$. Let $r_{1}, s_{1} \in R_{1}, r_{2}, s_{2} \in R_{2}, u=\left[\left(r_{1}, r_{2}\right),\left(s_{1}, s_{2}\right)\right]$. Therefore $d(u)=\left(0, d\left(\left[r_{2}, s_{2}\right]\right)\right)$ and $[d(u), u]=\left(0,\left[d\left(\left[r_{2}, s_{2}\right]\right),\left[r_{2}, s_{2}\right]\right]\right)$. Since $\left[d\left(\left[r_{2}, s_{2}\right]\right),\left[r_{2}, s_{2}\right]\right]^{2} \in Z\left(R_{2}\right)$, then

$$
[d(u), u]^{2}=\left(0,\left[d\left(\left[r_{2}, s_{2}\right]\right),\left[r_{2}, s_{2}\right]\right]\right)^{2}=\left(0,\left[d\left(\left[r_{2}, s_{2}\right]\right),\left[r_{2}, s_{2}\right]\right]^{2}\right) \in Z(R)
$$

but $R$ does not satisfy $s_{4}$.

The related object we need to mention is the left Utumi quotient ring $U$ of $R$. For basic definitions and preliminary results we refer the reader to [1], $[5],[9]$.

In order to prove the main result of this section we will make use of the following facts:

Claim 1 ([1, Proposition 2.5.1]). Any derivation of a semiprime ring $R$ can be uniquely extended to a derivation of its left Utumi quotient ring $U$, and so any derivation of $R$ can be defined on the whole $U$. 
Claim 2 ([3, p. 38]). If $R$ is semiprime then so is its left Utumi quotient ring. The extended centroid $C$ of a semiprime ring coincides with the center of its left Utumi quotient ring.

Claim 3 ([3, p. 42]). Let $B$ be the set of all the idempotents in $C$, the extended centroid of $R$. Assume $R$ is a B-algebra orthogonal complete. For any maximal ideal $P$ of $B, P R$ forms a minimal prime ideal of $R$, which is invariant under any derivation of $R$.

We will prove the following:

Theorem 2.1. Let $R$ be a 2-torsion free semiprime ring, $d$ a nonzero derivation of $R, n$ a fixed positive integer, $U$ the left Utumi quotient ring of $R$ and $[d([x, y]),[x, y]]^{n}=0$, for any $x, y \in R$. Then there exists a central idempotent element e of $U$ such that on the direct sum decomposition $e U \oplus$ $(1-e) U, d$ vanishes identically on $e U$ and the ring $(1-e) U$ is commutative.

Proof. Since $R$ is semiprime, by Claim 2, $Z(U)=C$, the extended centroid of $R$, and, by Claim 1 , the derivation $d$ can be uniquely extended on $U$. Since $U$ and $R$ satisfy the same differential identities (see [12]), then $[d([x, y]),[x, y]]^{n}=0$, for all $x, y \in U$. Let $B$ be the complete boolean algebra of idempotents in $C$ and $M$ be any maximal ideal of $B$.

Since $U$ is a $B$-algebra orthogonal complete (see [3, p. 42, (2) of Fact 1]), by Claim 3, $M U$ is a prime ideal of $U$, which is $d$-invariant. Denote $\bar{U}=U / M U$ and $\bar{d}$ the derivation induced by $d$ on $\bar{U}$. For any $\bar{x}, \bar{y} \in \bar{U}$, $[\bar{d}([\bar{x}, \bar{y}]),[\bar{x}, \bar{y}]]^{n}=0$. In particular $\bar{U}$ is a prime ring and so, by Lemma 1.1 , $\bar{d}=0$ in $\bar{U}$ or $\bar{U}$ is commutative. This implies that, for any maximal ideal $M$ of $B, d(U) \subseteq M U$ or $[U, U] \subseteq M U$. In any case $d(U)[U, U] \subseteq M U$, for all $M$. Therefore $d(U)[U, U] \subseteq \bigcap_{M} M U=0$.

By using the theory of orthogonal completion for semiprime rings (see [1, Chapter 3]), it follows that there exists a central idempotent element $e$ in $U$ such that on the direct sum decomposition $e U \oplus(1-e) U, d$ vanishes identically on $e U$ and the ring $(1-e) U$ is commutative.

We come now to our last result:

Theorem 2.2. Let $R$ be a 2-torsion free semiprime ring, $d$ a nonzero derivation of $R, n$ a fixed positive integer, $U$ the left Utumi quotient ring of $R$ and $[d([x, y]),[x, y]]^{n} \in Z(R)$, for any $x, y \in R$. Then there exists a central idempotent $e$ of $U$ such that, on the direct sum decomposition $U=$ $e U \oplus(1-e) U$, the derivation $d$ vanishes identically on $e U$ and the ring $(1-e) U$ satisfies $s_{4}$. 
Proof. By Claim 2, $Z(U)=C$, and by Claim $1 d$ can be uniquely defined on the whole $U$. Since $U$ and $R$ satisfy the same differential identities, then $[d([x, y]),[x, y]]^{n} \in C$, for all $x, y \in U$. Let $B$ be the complete boolean algebra of idempotents in $C$ and $M$ any maximal ideal of $B$. As already pointed out in the proof of Theorem 2.1, $U$ is a B-algebra orthogonal complete and by Claim 3, $M U$ is a prime ideal of $U$, which is d-invariant. Let $\bar{d}$ the derivation induced by $d$ on $\bar{U}=U / M U$. Since $Z(\bar{U})=(C+M U) / M U=C / M U$, then $[\bar{d}([x, y]),[x, y]]^{n} \in(C+M U) / M U$, for any $x, y \in \bar{U}$. Moreover $\bar{U}$ is a prime ring, hence we may conclude, by Theorem 1.1 , that $\bar{d}=0$ in $\bar{U}$ or $\bar{U}$ satisfies $s_{4}$. This implies that, for any maximal ideal $M$ of $B$, $d(U) \subseteq M U$ or $s_{4}\left(x_{1}, x_{2}, x_{3}, x_{4}\right) \subseteq M U$, for all $x_{1}, x_{2}, x_{3}, x_{4} \in U$. In any case $d(U) s_{4}\left(x_{1}, x_{2}, x_{3}, x_{4}\right) \subseteq \bigcap_{M} M U=0$. From [1, Chapter 3], there exists a central idempotent element $e$ of $U$, the left Utumi quotient ring of $R$, such that there exists a central idempotent $e$ of $U$ such that $d(e U)=0$ and $(1-e) U$ satisfies $s_{4}$.

\section{References}

[1] K.I. Beidar, W.S. Martindale and V. Mikhalev, Rings with generalized identities, Pure and Applied Math., Dekker, New York, 1996.

[2] C.L. Chuang, GPI's having coefficients in Utumi quotient rings, Proc. Amer. Math. Soc., 103(3) (1988), 723-728.

[3] _ Hypercentral derivations, J. Algebra, 166 (1994), 34-71.

[4] J.S. Erickson, W.S. Martindale III and J.M. Osborn, Prime nonassociative algebras, Pacific J. Math., 60 (1975), 49-63.

[5] C. Faith, Lecture on Injective Modules and Quotient Rings, Lecture Notes in Mathematics, 49, Springer Verlag, New York, 1967.

[6] I.N. Herstein, Topics in ring theory, Univ. Chicago Press, 1969.

[7] N. Jacobson, PI-algebras, an introduction, Lecture notes in Math., 441, Springer Verlag, New York, 1975.

[8] V.K. Kharchenko, Differential identities of prime rings, Algebra and Logic, 17 (1978), 155-168.

[9] J. Lambek, Lecture on Rings and Modules, Blaisdell Waltham, MA, 1966.

[10] C. Lanski, An Engel condition with derivation, Proc. Amer. Math. Soc., 118(3) (1993), 731-734.

[11] _ Differential identities, Lie ideals and Posner's theorems, Pacific J. Math., 134(2) (1988), 275-297.

[12] T.K. Lee, Semiprime rings with differential identities, Bull. Inst. Math. Acad. Sinica, 20(1) (1992), 27-38.

[13] W.S. Martindale III, Prime rings satisfying a generalized polynomial identity, J. Algebra, 12 (1969), 576-584. 
[14] E.C. Posner, Derivations in prime rings, Proc. Amer. Math. Soc., 8 (1957), 10931100 .

Received August 19, 1998 and revised November 20, 1998.

Dipartimento di Matematica ed Applicazioni

UNIVERSitÀ Di PALERMO

90123 PALERMO

ITALY

E-mail address: lcarini@dipmat.unime.it

Dipartimento di Matematica

UNIVERSitÀ Di MESSINA

98166 MESSINA

ITALY

E-mail address: enzo@dipmat.unime.it 